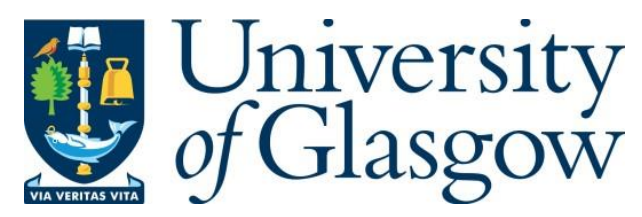

Sherazi, H. H. R., Imran, M. A., Boggia, G. and Grieco, L. A. (2018) Energy harvesting in LoRaWAN: a cost analysis for the industry 4.0. IEEE

Communications Letters, 22(11), pp. 2358-2361.

There may be differences between this version and the published version. You are advised to consult the publisher's version if you wish to cite from it.

http://eprints.gla.ac.uk/167687/

Deposited on: 31 August 2018

Enlighten - Research publications by members of the University of Glasgow

http://eprints.gla.ac.uk 


\title{
Energy Harvesting in LoRaWAN: A Cost Analysis for the Industry 4.0
}

\author{
Hafiz Husnain Raza Sherazi, Member, IEEE, Muhammad Ali Imran, Senior Member, IEEE, \\ Gennaro Boggia, Senior Member, IEEE and Luigi Alfredo Grieco, Senior Member, IEEE,
}

\begin{abstract}
Exploiting the advantages brought by long-range radio communications and extremely low power consumptions, LoRaWAN is capable to support low rate industry 4.0 services. Despite being energy-efficient, LoRa motes can still undergo frequent battery replenishments caused by the monitoring requirements of industrial applications. Duty-cycle constrained operations can partially face this issue at the expense of increased communication delays which, in turn, inflate higher costs due to damaged products on the production line. This letter proposes a model to analyze this cost tradeoff against different sensing intervals. It further highlights the impact of energy harvesting sources on this cost relationship mapping a way towards improved production efficiency.
\end{abstract}

Index Terms-Battery replacement cost, damage penalty, energy harvesting, Industrial IoT, Industry 4.0, LoRaWAN.

\section{INTRODUCTION}

The relentless expansion of today's Internet technology is driving automation processes towards the fourth industrial revolution. The essence of Industry $4.0[1]$ is the decentralized decision-making approach, based on real-time data collected through cyber-physical systems that constantly monitor the physical resources on the manufacturing line. These smart factories are able to suddenly react to unwanted events and avoid possible financial losses thanks to Predictive Maintenance (PM), Anomaly Detection, and Condition Monitoring systems.

Pervasive connectivity technologies are the key enablers of the Industry 4.0 paradigm. In this context, Low Power-Wide Area Network (LP-WAN) [2] are quickly gaining momentum because of their inherent capabilities to match coverage, scalability, and energy efficiency requirements of Industrial Internet of Things (IIoT) deployments. Many different LPWAN technologies are available nowadays including, but not limited to, LoRaWAN, Sigfox, NB-IoT, LTE-M1, Weightless, DASH7, and Ingenu [2].

Despite being extremely energy efficient, most of the enddevices belonging to LP-WAN are still battery-powered. Frequent battery replenishments can be required to maintain ongoing operations, which obviously raises operational costs. Several works (e.g., [3]- [6]) have previously been presented to

H. H. R. Sherazi, G. Boggia, and L. A. Grieco are with the Department of Electrical and Information Engineering (DEI), Politecnico di Bari, Bari, 70125 Italy. e-mail:\{sherazi, gennaro.boggia, alfredo.grieco \}@poliba.it.

M. A. Imran is with the School of Engineering, University of Glasgow, Glasgow, United Kingdom. e-mail: muhammad.imran@glasgow.ac.uk.

This research was partly funded by EPSRC Global Challenges Research Fund - the DARE project EP/P028764/1. prolong the battery life of sensor nodes employing different techniques but, their strategies neither take into account the peculiarities of industrial environments, nor they discuss the cost of achieved lifetime in terms of damage penalty and, consequently, the battery replacement cost. For example, a classic approach to further extend battery life is to reduce the duty-cycle [5], [6] of sensing devices, which inflates communication delays. Unfortunately, in some industrial plants (e.g., automotive industry), the timely detection of anomalies at various stages of the production process is the only way to avoid huge financial losses(i.e., huge damage penalty).

Facing the critical interplay between latency, battery lifetime, and the requirements of Industry 4.0 applications requires a novel design methodology that accounts for all the costs and benefits entailed by LP-WAN deployments in smart industrial environments.

To bridge this gap, the following contributions are provided hereby: first, a model is presented to evaluate the lifetime of LoRa monitoring nodes. Second, battery replacement and damage penalty costs are evaluated to study their mutual relationship. Third, the evaluation of renewable energy sources to feed LoRa nodes in industrial environments is considered and a cost-benefits analysis is proposed. Without lack of generality, this contribution will focus on the Long Range Wide Area Network (LoRaWAN) architecture, but the methodology developed hereby can be applied, with some customizations, to any LP-WAN technology. Thanks to the proposed methodology, optimal sensing intervals (and related costs) have been derived in both harvesting and non-energy harvesting scenarios. It is encouraging to note that the aggregated cost in the presence of harvested energy can be reduced to one-fifth of the overall cost in the non-energy harvesting environment.

\section{SYSTEM MODEL}

The LoRaWAN configuration settings considered hereby are presented in Table 1 . Being battery-powered, LoRa end-devices

TABLE I

LORAWAN ASSUMED PARAMETERS FOR THE LIFETIME EVALUATION

\begin{tabular}{lc}
\hline LoRaWAN Parameters & Values \\
\hline \hline Application Payload & $1-3 \mathrm{Bytes}$ \\
Modulation Technique & LoRa (based on CSS) \\
Spreading Factor (SF) & $7-12$ \\
Coding Rate & $4 / 5$ \\
Bandwidth & $125 \mathrm{kHz}$ \\
Preamble Symbols & 8 \\
Transmit Power & $14 \mathrm{dBm}$ \\
Distance b/w mote and the Gateway & $1200 \mathrm{~m}$ \\
\hline
\end{tabular}


are assumed to follow periodic transmission intervals to prolong their operations. The monitoring devices are put on sleep after each transmission interval until their next measurement. Let the pause time between two consecutive monitoring slots of a LoRaWAN device is sensing interval, $\Delta T_{\text {sense }}$, and $\Delta t_{s}$ be the duration when the end-device remains in sleep mode then, the sensing interval can be expressed as:

$$
\Delta T_{\text {sense }}=\Delta t_{s}+\Delta t_{\text {setup }} .
$$

where, $\Delta t_{\text {setup }}$ is the time required for a monitoring node to switch between active and sleep modes (i.e. 2. $\Delta t_{\text {switch }}$ ).

Several sensing intervals have been considered ranging from one to five minutes to investigate the impact of varying $\Delta T_{\text {sense }}$ on the energy consumption of LoRa monitoring devices. To this end, LoRa monitoring devices from Semtech Inc. are studied considering the current draw of their chipset as $44 \mathrm{~mA}$ when transmitting with $14 \mathrm{dBm}$ output power [5]. The monitoring devices are assumed to be the periodic transmitters only in a unidirectional way. They conventionally follow active and sleep modes where average current draws in sleep and switch mode $\left(\mu(I)_{s}\right.$ and $\left.\mu(I)_{s w}\right)$ are $100 \mathrm{nA}$ and $21.9 \mathrm{~mA}$, respectively [7].

Then, the mean charge, $\mu(Q)$, in each state (active, sleep, and switch) can be evaluated considering current draws of Semtech's LoRa monitoring node in different modes and time duration when a node stays in that state (e.g., $\mu(Q)_{T X}=$ $\mu(I)_{T X} \cdot \Delta t_{T X}$; where, $\mu(I)_{T X}$ is the average current draw of monitoring node in transmit mode and $\Delta t_{T X}$ is the duration of active period). The total charge, $\mu(Q)_{t o t}$, is the summation of the products of average current flow and the time duration in all possible states and can be seen as:

$$
\mu(Q)_{t o t}=\sum_{S} \mu(I)_{S} \cdot \Delta t_{S}, S \in\{T X, s, s w\}
$$

As the total mean energy, $\mu(E)_{t o t}$, is the product of total average charge and voltage applied on SX1272 end-device so it can be evaluated as:

$$
\mu(E)_{t o t}=\mu(Q)_{t o t} \cdot V_{S X 1272}
$$

Likewise, the average energy consumed per day, $\mu(E)_{d a y}$, and the average energy consumption during a whole year can also be calculated using Eq. (3). Now, the battery life (in years) is evaluated assuming the total battery capacity, $(\mathrm{Cap})_{\text {batt. }}$, of 1000 mAh (11880J@3.3V):

$$
\mu(\text { Life })_{b a t t .}=\frac{(\text { Cap })_{\text {batt. }}}{\mu(E)_{d a y}} \cdot 365
$$

\section{A. Battery replacement cost}

The battery replacement cost of LoRa monitoring nodes deployed throughout the production line is the first significant cost that is considered critically while designing the monitoring and control system. This cost can further be split into three types of costs; battery purchasing cost, installation cost, and the dispose-of cost for the old battery. The first one is taken as the fixed cost neglecting the inflation factor with time while the second one depends upon the type of industry and the replacement complexity of LoRa monitoring node whose battery needs replenishment. For example, the monitoring nodes installed internally inside a machinery would incur more labor cost due to the complexity of task as compared to the one installed on the relatively simpler spot. Like purchasing cost, the dispose-of cost can also be assumed as a fixed cost. A set of assumptions followed to evaluate the associated battery replacement cost is presented in Table II.

TABLE II

ASSUMED VALUES FOR CUMULATIVE BATTERY REPLACEMENT COST EVALUATION

\begin{tabular}{lc}
\hline Cost Parameters & $\begin{array}{c}\text { Assumed } \\
\text { Values }\end{array}$ \\
\hline \hline Average battery life calculated for LoRa monitoring & $0.10-5.14$ \\
device @ 14dBm transmitting power (years) & \\
Cost assumed per battery $(£), C_{b}$ & 3.7 \\
Time period considered (years), T & 20 \\
The number of batteries per node & 1 \\
Variable battery installation labor cost per node $(£), C_{r}$ & $3.5-10$ \\
Dispose-of cost per node in period T $(£)$ & 0.10 \\
\hline
\end{tabular}

Battery purchase cost, $C_{\text {batt. }}$, can be respected as the total cost of purchasing the required number of batteries in a time period and can be evaluated as:

$$
C_{\text {batt. }}=C_{b} \cdot N_{c y c}
$$

where, $C_{b}$ and $N_{c y c}$ are the cost incurred to purchase a single battery and number of replacement cycles required in a given time period, respectively. Here, it is important to note that a time period of 20 years is considered for this cost evaluation because it is believed to be the fair lifetime achievable through LoRa monitoring nodes in energy harvesting scenarios. Similarly, cumulative installation cost, $C_{\text {inst. }}$, is the variable labor cost that can be calculated as:

$$
C_{\text {inst. }}=C_{r} \cdot N_{c y c}
$$

where, $C_{r}$ is the variable replacement labor cost per node depending upon the complexity of spot. The battery disposeof cost, $C_{d i s s .}$, is the cost incurred on disposing-of the replaced batteries that is not usually higher but it may still be significant in case of large-scale network deployment where thousands of nodes need replacement in a time period. $C_{d i s s}$. is calculated assuming $£ 1400$ as the average dispose-of cost per ton of battery wastage from the recent statistics published by UK Government authorities [8]. Hence, the total battery replacement cost in pounds, $C_{t o t}$, can be seen as the summation of these costs in a time period of twenty years for LoRa monitoring node in the system, and can be expressed as:

$$
C_{t o t}=\sum_{s} C_{s}, s \in\{\text { batt., inst., diss. }\}
$$

\section{B. Damage penalty}

The damage penalty can be referred as the cost of damaged products on the production line due to a possible delay in anomaly detection. This delay can be seen as damage interval, $\Delta T_{d a m}$. and expressed as:

$$
\Delta T_{\text {dam. }}=t_{d}-t_{o} ; 0 \leqslant \Delta T_{\text {dam. }} \leqslant \Delta T_{\text {sense }}
$$

where, $t_{d}$ and $t_{o}$ are the anomaly detection time and anomaly occurrence time, respectively. Let $P_{d a m}$. be the damage penalty and $R_{\text {prod. }}$ be the rate of production at the production 
TABLE III

ASSUMED ENERGY HARVESTING SOURCES WITH THEIR AVERAGE POTENTIAL [9]

\begin{tabular}{|c|c|c|}
\hline Energy Harvesting Source & Assumptions & $\begin{array}{c}\text { Harvesting } \\
\text { Potential }(\mathbf{J})\end{array}$ \\
\hline $\begin{array}{l}\text { Photoelectric (artificial light } \\
\text { sources) }\end{array}$ & $\begin{array}{l}\text { Average of office hours (8 } \\
\text { hours @2001x) }\end{array}$ & 4.3 \\
\hline $\begin{array}{l}\text { Thermoelectric (internal } \\
\text { and external heat difference) }\end{array}$ & $\begin{array}{l}10 \text { hour@ } 5^{\circ} \mathrm{C} \text { and } 5 \text { hours } \\
@ 10^{\circ} \mathrm{C}\end{array}$ & 6.2 \\
\hline $\begin{array}{l}\text { RF Energy (radio signals } \\
\text { within the plant) }\end{array}$ & $\begin{array}{l}3 \mathrm{~W} \text { transmitted through } 5 \mathrm{~m} \\
\text { distant source @ } 9 \mathrm{MHz}\end{array}$ & 1.8 \\
\hline $\begin{array}{l}\text { The total amount of har- } \\
\text { vested energy }\end{array}$ & $\begin{array}{l}\text { Considering three different } \\
\text { sources }\end{array}$ & 12.3 \\
\hline
\end{tabular}

line (taken in terms of the number of products manufactured per minute), then the damage penalty can be calculated as:

$$
P_{\text {dam. }}=\Delta T_{\text {dam. }} \times R_{\text {prod. }} \times C_{\text {unit }}
$$

where, $C_{\text {unit }}$ is the unit cost of production assumed for a specific unfinished product. As the domain of damage interval is increased with increasing value of $\Delta T_{\text {sense, }}$, the damage penalty also keeps on increasing. The damage penalty is evaluated for different categories of products like cheap, medium, expensive, and very expensive where per unit production cost is supposed as $10,70,150$, and 500 while the rate of manufactured products per minute is $30,6,3$, and 1 , respectively.

\section{ENERGY HARVESTING FOR INDUSTRIAL MONITORING}

Energy harvested from renewable energy sources, available in the industrial environment, can play its role to improve both performance and production efficiency in the industry. For making the evaluation as simple as possible, an average harvesting potential per day for three different renewable energy sources is considered in this study (see Table III). This potential for harvesting energy is commonly available in most of the industrial environments and has already been exploited by industrial monitoring applications reported in [9], [10].

\section{A. Battery life with energy harvesting}

Let $S=\left\{e_{1}, e_{2}, \ldots, e_{n}\right\}$ be the amount of harvested energy being added to the system through $n$ different renewable energy sources where, $n \in \mathbb{N}$, then the energy available in the buffer can be represented as:

$$
e^{b}=\sum_{s=1}^{n} e_{s}
$$

Now, dividing the total harvesting time into $K$ different slots $\{1,2,3, \ldots, \mathrm{k}-1, \mathrm{k}\} \mid k \in \mathbb{R}$, the amount of harvested energy available within the energy buffer at the end of slot $\mathrm{k}$ can be expressed as $e_{k}^{b}=\left(e_{k-1}^{b}-e_{k}^{i}\right)+e_{k}^{h}$; where, $e_{k-1}^{b}$ is the available energy in the buffer until the previous slot, $e_{k}^{i}$ is the amount of instantaneous energy consumed during current slot $k$, and $e_{k}^{h}$ is the newly harvested energy added to the system in current slot $k$.

Here, the amount of harvested energy over all $k$ slots can be expressed as follows realizing the above expression:

$$
\int_{0}^{k} E^{b} d k=\left[\int_{0}^{k-1} e^{b} d k-\int_{0}^{k} e^{i} d k\right]+\int_{0}^{k} e^{h} d k
$$

given that $\int_{0}^{k-1} e^{b} d k>\int_{0}^{k} e^{i} d k$ for an uninterrupted operation. This is the amount of energy left in the energy buffer after slot $k$. Hence, substituting the value of $e^{b}$ from Eq. 10 in Eq. 11:

$\int_{0}^{k} E^{b} d k=\left[\sum_{s=1}^{n} \int_{0}^{k-1} e_{s}^{b} d k-\sum_{s=1}^{n} \int_{0}^{k} e_{s}^{i} d k\right]+\sum_{s=1}^{n} \int_{0}^{k} e_{s}^{h} d k$

If there are $k$ slots in a day, then the amount of energy harvested per day, $E_{\text {day }}^{h}$, is equal to the amount of energy added to the system over $\mathrm{k}$ time slots as follows:

$$
\mu\left(E^{h}\right)_{d a y}=\int_{0}^{k} E^{b} d k
$$

Now, realizing the above equation, we can rewrite the Eq. 12 as:

$\mu\left(E^{h}\right)_{d a y}=\left[\sum_{s=1}^{n} \int_{0}^{k-1} e_{s}^{b} d k-\sum_{s=1}^{n} \int_{0}^{k} e_{s}^{i} d k\right]+\sum_{s=1}^{n} \int_{0}^{k} e_{s}^{h} d k$

Hence, the new energy requirement per day, $\mu\left(E^{\prime}\right)_{d a y}$, can easily be evaluated as a difference of previous energy demand drawn per day from Eq. (3), $\mu(E)_{d a y}$, and the newly harvested energy per day, $\mu(E)_{d a y}^{h}$, and can be represented as:

$$
\mu\left(E^{\prime}\right)_{d a y}=\mu(E)_{d a y}-\mu(E)_{d a y}^{h}
$$

Once the new energy requirement per day has been established, the new battery life of LoRa monitoring nodes can be evaluated employing Eq. (4) following the same set of assumptions regarding battery capacity and applied voltage $(1000 m A h @ 3.3 \mathrm{~V})$ as in non-energy harvesting life calculations. This new life would also lead to significant reduction in total battery replacement cost, $C_{t o t}$, without affecting the damage penalty.

\section{B. Sensing interval with energy harvesting}

Similarly, sensing interval can also be contracted in the presence of energy harvesting sources without compromising on the existing battery life. The extent of this contraction can rightly be assumed equal to the relaxation in energy quota due to harvested energy. It is the ratio of the average harvested energy per day to the previous energy demand per day. Hence, the new sensing interval in presence of energy harvesting sources, $\Delta T_{\text {sense }}^{\prime}$, can be expressed as:

$$
\Delta T_{\text {sense }}^{\prime}=\Delta T_{\text {sense }}-\left(\Delta T_{\text {sense }} \cdot \frac{\mu(E)_{\text {day }}^{h}}{\mu\left(E^{\prime}\right)_{\text {day }}}\right)
$$

The new sensing interval would serve to significantly reduce the damage penalty, $P_{d a m}$, while fixing the battery replacement cost.

\section{RESUlts AND Discussions}

This section spans the analysis for the above discussed costs following the model proposed in Section II.

With reference to battery life, it is worth mentioning that the battery replacement cost and the damage penalty both are critical, however, in an attempt to cut down the one, the other may tend to go higher and vice versa in non-energy 


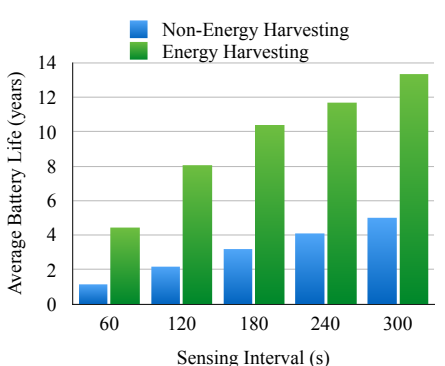

(a) Average battery life

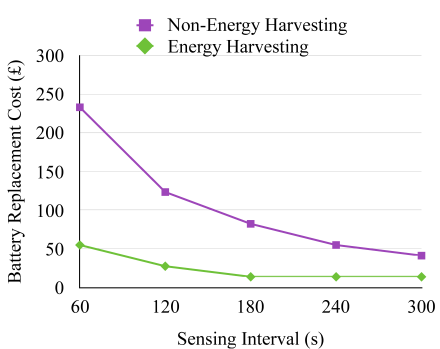

(b) Battery replacement cost
Fig. 1. Average battery life and battery replacement cost comparison between $\mathrm{EH}$ and non-EH environment against sensing interval

harvesting environment. On the other hand, the harvesting potential within a smart industry can dramatically make the difference as shown in Fig. 1(a) where the surplus amount of energy is capable to prolong the battery life many folds as we move along the sensing interval from $1 \mathrm{~min}$ to $5 \mathrm{~min}$. The new battery life would obviously be contributing towards cutting down the battery replacement costs as depicted in Fig. 1(b) The battery replacement cost can be maintained as low as just over $£ 13$ beyond the sensing interval over $3 \mathrm{~min}$. The battery replacement cost goes on increasing when we go on shrinking the sensing interval because more number of battery replacement cycles are needed when the monitoring nodes wake up more frequently (e.g., in case of $1 \mathrm{~min}$ sensing interval). Similarly, replacement cost does not go beyond $£ 50$ in energy harvesting scenario even if we sense every minute.

Concerning sensing intervals, the industries with a significantly higher unit cost of production, $C_{u n i t}$, may consider saving on the damage penalty as compared to the battery replacement costs. The expert systems within these industries need frequent data collection for generating more updated alerts aiming at reporting the anomaly as early as possible. Having this in mind, a new sensing interval can also be derived instead of prolonging the battery life in compliance with the extra energy quota available through harvested energy. This way, it is possible to confine the damage penalty up to just over thousand pounds beyond sensing interval of $85 \mathrm{~s}$ even in the most expensive category (where, $C_{\text {unit }}=£ 500$ ) as seen in Fig. 2(a). It can further be reduced up to $50 \%$ in an industry with low $C_{\text {unit }}$. Fig. 2(b) shows the rate of interval contraction and the impact of contracting the sensing interval on the overall cost picture. It argues that no linear increase in the overall cost picture is evident moving along the sensing interval as compared to non-energy harvesting environment. Interestingly, the contraction rate goes on so higher that the overall cost starts going down. The right most bar of Fig. 2(b) depicts that the aggregated costs recorded on $\Delta T_{\text {sense }}=6 \mathrm{~min}$ are even better (i.e. lower) than the aggregate costs recorded on $\Delta T_{\text {sense }}=1 \mathrm{~min}$ which justifies the choice of fairly long sensing interval.

\section{CONCLUSION}

The work presents a model to get an insight of the interesting cost relationship for battery replacement and damage

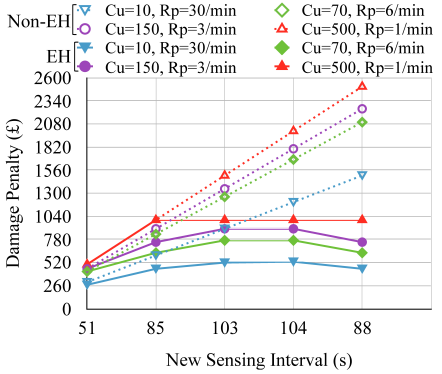

(a) Demage Penalty

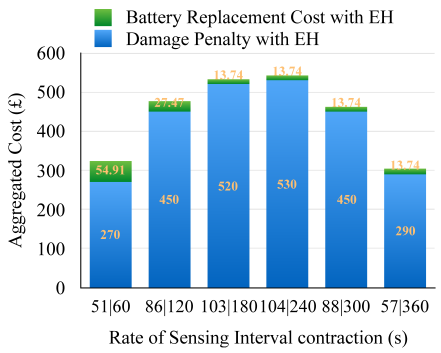

(b) Aggregate cost
Fig. 2. Damage penalty against new sensing interval and aggregate cost in energy harvesting environment against the rate of sensing interval contraction

penalty in industry 4.0, and highlights the following important aspects. First, the damage penalty remains higher than battery replacement cost for longer sensing intervals and both the costs tend to equalize around the sensing interval of $1 \mathrm{~min}$. Second, the battery replacement costs are decreased because of prolonged battery life in the presence of harvested energy without affecting the sensing interval. Third, renewable energy being added to the system also provides the flexibility to contract the sensing interval to achieve more updated alerts. Fourth, both the costs can be cut-down in case of harvested energy to get the least aggregated cost as compared to nonenergy harvesting environment. Fifth, the overall cost no more shows a linear increase in the presence of harvested energy and tends to decline beyond the sensing interval of $4 \mathrm{~min}$. It falls down to as lower as $£ 300$ especially on sensing interval of 6 min, even lower than the total cost noted on 1 min sensing interval.

\section{REFERENCES}

[1] Y. Liao, F. Deschamps, E. de Freitas Rocha Loures, and L. F. P. Ramos, "Past, present and future of industry 4.0 - a systematic literature review and research agenda proposal," International Journal of Production Research, vol. 55, no. 12, pp. 3609-3629, 2017.

[2] U. Raza, P. Kulkarni, and M. Sooriyabandara, "Low power wide area networks: An overview," IEEE Communications Surveys Tutorials, vol. 19, no. 2, pp. 855-873, Secondquarter 2017.

[3] A.-A. A. Boulogeorgos, P. D. Diamantoulakis, and G. K. Karagiannidis, "Low power wide area networks (lpwans) for internet of things (iot) applications: Research challenges and future trends," CoRR, vol abs/1611.07449, 2016.

[4] Y. Song, J. Lin, M. Tang, and S. Dong, "An internet of energy things based on wireless lpwan," Engineering, vol. 3, no. 4, pp. 460 - 466, 2017.

[5] C. Tunc and N. Akar, "Markov fluid queue model of an energy harvesting iot device with adaptive sensing," Performance Evaluation, vol. 111 , pp. 1 - 16, 2017.

[6] N. Michelusi and M. Levorato, "Energy-based adaptive multiple access in lpwan iot systems with energy harvesting," in Proc. of 2017 IEEE ISIT Symposium, June 2017, pp. 1112-1116.

[7] S. Inc., "Low power long range transceiver, sx1272/73 datasheet," Nov. 2015, [Revised Mar. 2017]. [Online]. Available: https://www.semtech. com/uploads/documents/sx1272.pdf

[8] "Impact Assessment Report (DEFRA1784)," Department for Environment, Food and Rural Affairs, Tech. Rep., March 2018.

[9] F. Touati, A. Galli, D. Crescini, P. Crescini, and A. B. Mnaouer, "Feasibility of air quality monitoring systems based on environmental energy harvesting," in Proc. of 2015 IEEE I2MTC Conference, May 2015, pp. 266-271.

[10] X. Lu, P. Wang, D. Niyato, D. I. Kim, and Z. Han, "Wireless networks with rf energy harvesting: A contemporary survey," IEEE Communications Surveys \& Tutorials, vol. 17, no. 2, pp. 757-789, 2015. 